OPEN ACCESS

Edited by:

Brijesh Kumar Singh,

Duke-NUS Medical School, Singapore

Reviewed by:

Patricia Silveyra,

Indiana University Bloomington,

United States

Prem Prakash Tripathi,

Indian Institute of Chemical Biology

(CSIR), India

*Correspondence:

Li-Peng Shi

shilipeng0206@163.com

Wen-Fu Cao

caowenfu9316@163.com

Specialty section:

This article was submitted to Respiratory Pharmacology,

a section of the journal

Frontiers in Pharmacology

Received: 12 May 2021

Accepted: 18 August 2021 Published: 15 September 2021

Citation:

DuX-Q, Shi L-P, Cao W-F, Chen Z-W,

Zuo $B$ and $H u J-Y$ (2021) Add-On

Effect of Honeysuckle in the Treatment

of Coronavirus Disease 2019: A

Systematic Review and Meta-Analysis.

Front. Pharmacol. 12:708636.

doi: $10.3389 /$ fphar.2021.708636

\section{Add-On Effect of Honeysuckle in the Treatment of Coronavirus Disease 2019: A Systematic Review and Meta-Analysis}

\author{
Xu-Qin Du ${ }^{1,2}$, Li-Peng Shi ${ }^{1,2 *}$, Wen-Fu Cao ${ }^{1,2,3 *}$, Zhi-Wei Chen ${ }^{1,2}$, Biao Zuo ${ }^{1,2}$ and \\ Jin-Yuan $\mathrm{Hu}^{1,2}$ \\ ${ }^{1}$ College of Traditional Chinese Medicine, Chongqing Medical University, Chongqing, China, ${ }^{2}$ Chongqing Key Laboratory of \\ Traditional Chinese Medicine for Prevention and Cure of Metabolic Diseases, Chongqing, China, ${ }^{3}$ Department of Chinese \\ Traditional Medicine, The First Affiliated Hospital of Chongqing Medical University, Chongqing, China
}

Background: The outbreak of coronavirus disease 2019 (COVID-19) has rapidly spread to become a global emergency since December 2019. Chinese herbal medicine plays an important role in the treatment of COVID-19. Chinese herbal medicine honeysuckle is an extremely used traditional edible and medicinal herb. Many trials suggest that honeysuckle has obtained a good curative effect for COVID-19; however, no systematic evaluation on the clinical efficacy of honeysuckle in the treatment of COVID-19 is reported. This study aimed to evaluate the efficacy and safety of Chinese herbal medicine honeysuckle in the treatment of COVID-19.

Methods: Seven electronic databases (PubMed, EMBASE, Cochrane Library, China National Knowledge Infrastructure, China Science and Technology Journal Database, Wanfang Database, and China Biology Medicine) were searched to identify randomized controlled trials (RCTs) of honeysuckle for adult patients (aged $\geq 18$ years) with COVID-19. The Cochrane Risk of Bias Tool was applied to assess the methodological quality of trials. Review Manager 5.3 software was used for data analysis.

Results: Overall, nine RCTs involving 1,286 patients were enrolled. Our meta-analyses found that combination therapy of honeysuckle and conventional therapy was more effective than conventional therapy alone in lung computed tomography (CT) [relative risk $(R R)=1.24,95 \%$ confidence interval $(95 \% \mathrm{Cl})(1.12,1.37), P<0.0001]$, clinical cure rate $[\mathrm{RR}=1.21,95 \% \mathrm{Cl}(1.12,1.31), P<0.00001]$, and rate of conversion to severe cases $[\mathrm{RR}=0.50,95 \% \mathrm{Cl}(0.33,0.76), P=0.001]$. Besides, combination therapy can improve the symptom score of fever, cough reduction rate, symptom score of cough, and inflammatory biomarkers (white blood cell (WBC) count; C-reactive protein (CRP)) $(P<0.05)$.

Conclusion: Honeysuckle combined with conventional therapy may be beneficial for the treatment of COVID-19 in improving lung CT, clinical cure rate, clinical symptoms, and laboratory indicators and reducing the rate of conversion to severe cases. Besides, combination therapy did not increase adverse drug events. More high-quality RCTs are needed in the future.

Keywords: coronavirus disease 2019, honeysuckle, systematic review, meta-analysis, randomized controlled trials 


\section{INTRODUCTION}

Since December 2019, coronavirus disease 2019 (COVID-19) caused by severe acute respiratory syndrome coronavirus 2 (SARS-CoV-2) has become a public health emergency of global concern (Sattler et al., 2020). As of May 6, 2021, more than 154.815 million confirmed cases and more than 3.236 million deaths had been reported globally (World Health Organization (WHO), 2021). Thus, there is an urgent need to prevent and treat COVID-19.

Through a series of prevention and medical treatment measures, the COVID-19 epidemic in China has been effectively controlled by May 6, 2021, with 103,731 confirmed cases and 98,392 cured cases (World Health Organization (WHO), 2021; National Health Commission of the people's Republic of China, 2021). Chinese herbal medicine plays an important role in the treatment of COVID-19 in view of no specific drugs approved for COVID-19. Chinese herbal medicine honeysuckle is an extremely used traditional edible-medicinal herb (Li et al., 2021). Pharmacological studies have already proved honeysuckle's ideal clinical therapeutic effects on inflammation and infectious diseases (Li et al., 2021). Also, it is reported that honeysuckle can effectively alleviate clinical symptoms of COVID-19 (Hu et al., 2020; Zhang et al., 2020) and inhibit SARS-CoV-2 replication (Zhou et al., 2020).

At present, there are only few trials on the treatment of COVID-19 with honeysuckle (Hu et al., 2020; Zhang et al., 2020), but many trials on the treatment of COVID-19 used Chinese herbal medicine including honeysuckle as the main components (Ai et al., 2020; Ding et al., 2020; Duan et al., 2020). These trials suggest that honeysuckle has obtained a good curative effect for COVID-19 (Hu et al., 2020; Zhang et al., 2020). Presently, there is no systematic evaluation report on the clinical efficacy of honeysuckle in the treatment of COVID-19. This review aimed to critically evaluate the effectiveness and safety of honeysuckle for COVID-19.

\section{METHODS}

The preferred reporting item for systematic review and metaanalysis (PRISMA) Evaluation Scale was used for reporting the results of this review (Moher et al., 2009). The protocol for this review is available in PROSPERO (https://www.crd.york.ac.uk/ prospero/, registration number is CRD42020224312).

\section{Database and Search Strategies}

The following seven databases were retrieved, including PubMed, EMBASE, Cochrane Library, China National Knowledge Infrastructure (CNKI), China Science and Technology Journal Database (VIP), Wanfang Database, and China Biology Medicine (CBM), from December 2019 to May 2021. There was no language restriction. The grouped keywords used as search strategy were as follows: "traditional Chinese medicine" OR "Chinese herbal medicine" OR "honeysuckle" OR "lonicera" OR “jinyinhua”) AND (“coronavirus disease 2019" OR "COVID-19" OR "novel coronavirus pneumonia” OR "SARS-
CoV-2") AND ("clinical trial" OR "randomized controlled trial" OR "randomised controlled trial" OR "lin chuang yan jiu"). The grouped keywords could be modified according to different databases.

Potentially eligible trials were obtained by manually searching the reference lists of published reviews and meta-analyses. We also retrieved the unpublished papers on honeysuckle for COVID-19.

\section{Inclusion and Exclusion Criteria}

We considered the following inclusion criteria: 1) study design: randomized controlled trials (RCTs); 2) participants: adult patients (aged $\geq 18$ years) diagnosed with COVID-19; the diagnostic criteria of COVID-19 refer to "Diagnosis and Treatment Guideline for COVID-19 (Trial 8th Edition) " (National Health Commission of the people's Republic of China, 2020); 3) interventions: patients in the treatment group were treated with honeysuckle alone or a combination treatment of honeysuckle and controls; the dose of honeysuckle was 5-30 g, along with a duration range of 5-15 days; the form and dosage of honeysuckle were included in the study description; 4) control: patients in the control group were treated by any conventional therapy or placebo; 5) outcomes: lung computed tomography (CT) was the primary outcome. High-resolution CT was used to observe changes in the lung field before and after treatment. The secondary outcomes included clinical cure rate, viral nucleic acid testing, rate of conversion to severe cases, clinical symptoms (e.g., fever, cough, and fatigue), inflammatory biomarkers [e.g., white blood cell (WBC) count, lymphocyte (LYM) count, and C-reactive protein (CRP)], and adverse drug events (e.g., adverse events rate, diarrhea, and liver damage).

We considered the following exclusion criteria: 1) study design: non-RCTs, such as retrospective studies, observational studies, case reports, and cross-over studies; non-RCTs were excluded due to potential high risk of bias and confounding; 2) participants: patients with a suspected diagnosis of COVID-19; 3) repeated data studies; 4) reviews.

Two reviewers independently screened the trials from seven databases according to the eligibility criteria. Duplicate publications were removed. Through reading the title, abstract, and full text, reviewers excluded the non-RCTs and irrelevant trials. Two reviewers independently extracted data according to the eligibility criteria. Any disagreements were consulted by a third reviewer.

\section{Data Collection}

The following information was documented in the data extraction tables: basic characteristics (e.g., the title, first authors' name, publication date, intervention schedule of treatment and control groups, and treatment duration), participant characteristics (e.g., age, gender, and sample size), outcome measures, and adverse drug events.

\section{Quality Assessment}

The methodological quality was independently evaluated by two reviewers according to the Cochrane Collaboration's tool (Higgins and Green, 2014). There were seven items of risk of bias (ROB): random sequence generation, allocation 


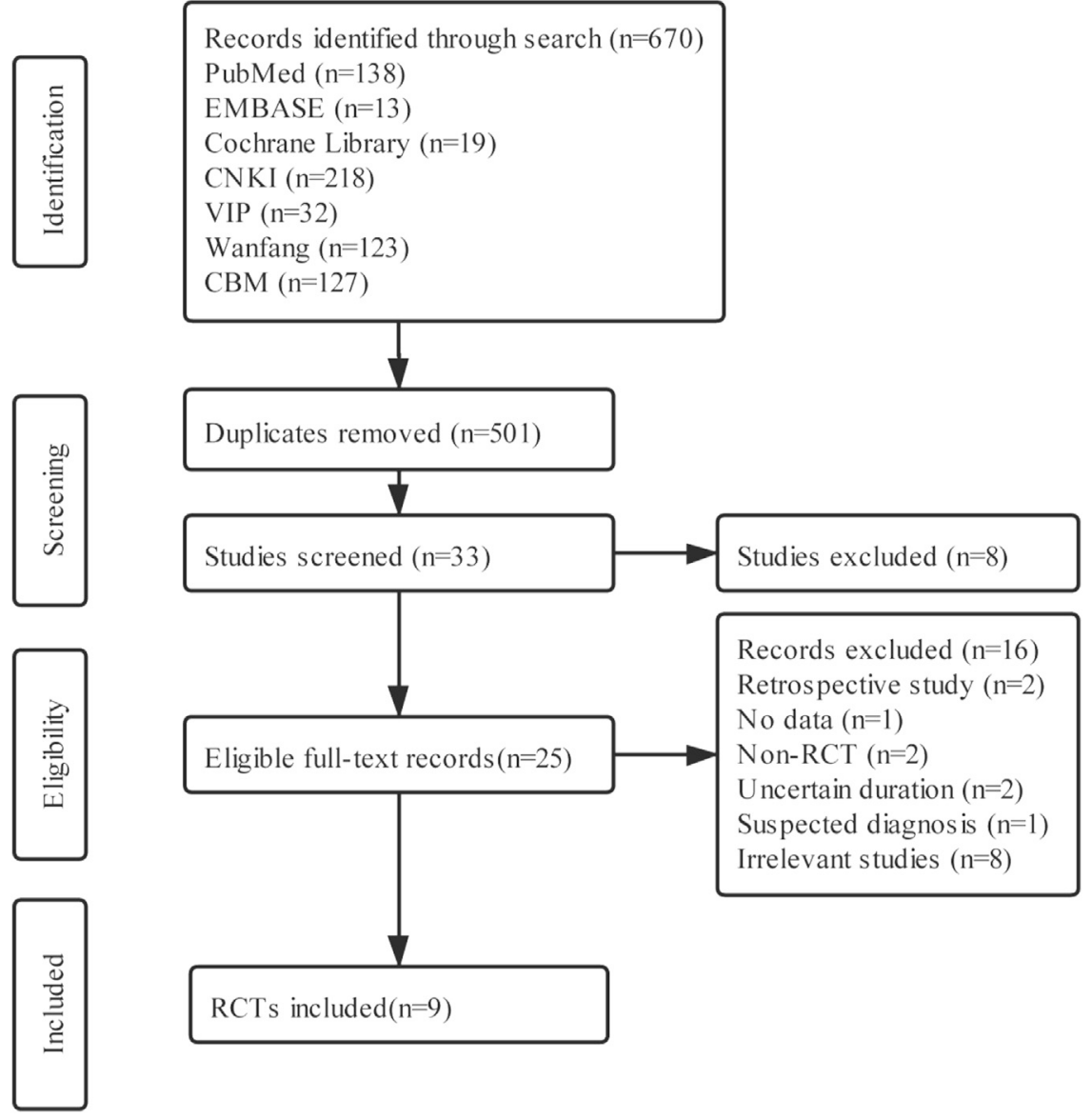

FIGURE 1 | The flowchart of study selection.

concealment, blinding of participants and personnel, blinding of outcome assessment, incomplete outcome data, selective reporting, and other biases. Each item was assessed at low $\mathrm{ROB}$, high $\mathrm{ROB}$, or unclear ROB. Any disagreements between reviewers were resolved by consultation with a third reviewer.

\section{Statistical Analysis}

Review Manager 5.3 software (the Cochrane Collaboration, 2014) was used to perform the quantitative synthesis. Relative risk (RR) was used for the dichotomous variables. Mean difference (MD) or standard mean difference (SMD) was used for the continuous variables. Confidence intervals (CIs) were set as $95 \%$. Heterogeneity was tested with the $\chi^{2}$ test and the $I^{2}$ statistical value. Assuming that the $p$-value from the $\chi 2$ test was more than 0.10 or $I^{2} \leq 50 \%$, a fixed-effect model was used to assess the differences between two groups; otherwise, a random-effects model was applied. A subgroup analysis of the primary outcome was performed according to the clinical classification of COVID-19. Subgroup analyses of viral nucleic acid testing and rate of conversion to severe cases were performed according to the Chinese herbal medicine component. Sensitivity analysis was conducted by the leave-one-out method (Panahi et al., 2015). When the number of trials on an outcome measure was larger than ten, a funnel plot analysis was performed to evaluate the reporting bias (Liu et al., 2020). Statistically significant results were defined by $p<0.05$.

\section{RESULTS}

\section{Study Selection}

The flowchart of study selection is shown in (Figure 1). A total of nine eligible trials were included (Fu et al., 2020a; Ai et al., 2020; Ding et al., 2020; Duan et al., 2020; Fu et al., 2020b; Hu et al., 2020; Yu et al., 2020; Zhang et al., 2020; Hu et al., 2021). One article was published online in English (Hu et al., 2021), and the rest were reported online in Chinese.

\section{Study Characteristics}

The characteristics of included trials are listed in Table 1. All the trials were conducted in China in 2020, among them, two studies were multicentered trials (Hu et al., 2020; Hu et al., 2021) and seven were single-centered trials (Ai et al., 2020; Ding et al., 2020; Duan et al., 2020; Hu et al., 2020; Yu et al., 2020; Zhang et al., 2020; Hu et al., 2021). The sample size of the included trials ranged from 65 to 295 (total 1,286). The treatment duration ranged from 5 to 15 days. Patients in the treatment group were treated with combination 
TABLE 1 | The characteristics of included trials.

\begin{tabular}{|c|c|c|c|c|c|c|c|}
\hline $\begin{array}{l}\text { First } \\
\text { author }\end{array}$ & $\begin{array}{l}\text { Type of } \\
\text { COVID-19 }\end{array}$ & $\begin{array}{c}\text { Sample } \\
\text { size (M/F) }\end{array}$ & Age (yrs) & Intervention & Control & Duration & Outcome measures \\
\hline $\begin{array}{l}\text { Ai et al. } \\
(2020)\end{array}$ & Nonsevere & $\begin{array}{l}\mathrm{T}: 55(24 / \\
31) \mathrm{C}: 43 \\
(17 / 26)\end{array}$ & $\mathrm{T}: 57.4 \pm 21.3 \mathrm{C}: 50.8 \pm 23.5$ & $\begin{array}{l}\text { Pneumonia No.1 } \\
\text { formula ( } 1 \text { dose/d) } \\
+ \text { conventional } \\
\text { therapy }\end{array}$ & $\begin{array}{l}\text { Conventional therapy } \\
\text { including arbidol, lopinavir/ } \\
\text { tonavir, chloroquine, and } \\
\text { symptomatic treatment }\end{array}$ & 12 days & $\begin{array}{l}\text { Clinical cure rate, } \\
\text { inflammatory biomarkers, } \\
\text { and adverse events }\end{array}$ \\
\hline $\begin{array}{l}\text { Ding } \\
\text { et al. } \\
(2020)\end{array}$ & $\begin{array}{l}\text { Mild/ } \\
\text { moderate/ } \\
\text { severe }\end{array}$ & $\begin{array}{l}\mathrm{T}: 51(39 / \\
12) \mathrm{C}: 49 \\
(39 / 10)\end{array}$ & $\mathrm{T}: 57.4 \pm 21.3 \mathrm{C}: 50.8 \pm 23.5$ & $\begin{array}{l}\text { Qingfei Touxie } \\
\text { Fuzheng recipe } \\
(1 \text { dose/d) }+ \\
\text { conventional } \\
\text { therapy }\end{array}$ & $\begin{array}{l}\text { Conventional therapy } \\
\text { including interferon- } \alpha \text {, } \\
\text { ribavirin, quinolones and/or } \\
\text { third-generation } \\
\text { cephalosporins, and } \\
\text { symptomatic treatment }\end{array}$ & 10 days & $\begin{array}{l}\text { Lung CT, clinical } \\
\text { symptoms, inflammatory } \\
\text { biomarkers, and adverse } \\
\text { events }\end{array}$ \\
\hline $\begin{array}{l}\text { Duan } \\
\text { et al. } \\
(2020)\end{array}$ & Mild & $\begin{array}{c}\mathrm{T}: 82(39 / \\
43) \mathrm{C}: 41 \\
(23 / 18)\end{array}$ & $\mathrm{T}: 51.99 \pm 13.88 \mathrm{C}: 50.29 \pm 13.17$ & $\begin{array}{l}\text { Jinhua Qinggan } \\
\text { granule ( } 1 \text { dose/ } \\
\text { time, tid) }+ \\
\text { conventional } \\
\text { therapy }\end{array}$ & $\begin{array}{l}\text { Conventional therapy } \\
\text { including antiviral, anti- } \\
\text { infection, and other } \\
\text { symptomatic treatments }\end{array}$ & 5 days & $\begin{array}{l}\text { Clinical symptoms and } \\
\text { adverse events }\end{array}$ \\
\hline $\begin{array}{l}\text { Fu et al. } \\
\text { (2020a) }\end{array}$ & $\begin{array}{l}\text { Mild/ } \\
\text { moderate }\end{array}$ & $\begin{array}{l}\mathrm{T}: 32(17 / \\
15) \mathrm{C}: 33 \\
(19 / 14)\end{array}$ & $\mathrm{T}: 43.26 \pm 7.15 \mathrm{C}: 43.68 \pm 6.45$ & $\begin{array}{l}\text { Toujie Quwen } \\
\text { granule ( } 1 \text { dose/ } \\
\text { time, bid) }+ \\
\text { conventional } \\
\text { therapy }\end{array}$ & $\begin{array}{l}\text { Conventional therapy } \\
\text { including abidor tablets, } \\
\text { moxifloxacin tablets, and } \\
\text { ambroxol tablets }\end{array}$ & 10 days & $\begin{array}{l}\text { Lung } \mathrm{CT} \text {, clinical cure } \\
\text { rate, rate of conversion to } \\
\text { severe cases, clinical } \\
\text { symptoms, inflammatory } \\
\text { biomarkers, and adverse } \\
\text { events }\end{array}$ \\
\hline $\begin{array}{l}\text { Fu et al. } \\
\text { (2020b) }\end{array}$ & Moderate & $\begin{array}{l}\mathrm{T}: 37(19 / \\
18) \mathrm{C}: 36 \\
(19 / 17)\end{array}$ & $\mathrm{T}: 45.26 \pm 7.25 \mathrm{C}: 44.68 \pm 7.45$ & $\begin{array}{l}\text { Toujie Quwen } \\
\text { granule ( } 1 \text { dose/ } \\
\text { time, bid) }+ \\
\text { conventional } \\
\text { therapy }\end{array}$ & $\begin{array}{l}\text { Conventional therapy } \\
\text { including abidor tablets and } \\
\text { ambroxol tablets }\end{array}$ & 15 days & $\begin{array}{l}\text { Clinical cure rate, rate of } \\
\text { conversion to severe } \\
\text { cases, clinical symptoms, } \\
\text { inflammatory biomarkers, } \\
\text { and adverse events }\end{array}$ \\
\hline $\begin{array}{l}\text { Hu et al. } \\
(2020)\end{array}$ & Moderate & $\begin{array}{c}\mathrm{T}: 100(49 / \\
51) \mathrm{C}: 100 \\
(55 / 45)\end{array}$ & $\mathrm{T}: 47.00 \pm 14.06 \mathrm{C}: 49.28 \pm 11.14$ & $\begin{array}{l}\text { Jinyinhua oral liquid } \\
(120 \mathrm{ml} / \text { time, tid })+ \\
\text { conventional } \\
\text { therapy }\end{array}$ & $\begin{array}{l}\text { Conventional therapy } \\
\text { including interferon- } \alpha \text {, } \\
\text { lopinavir/tonavir tablets, and } \\
\text { symptomatic treatment }\end{array}$ & 10 days & $\begin{array}{l}\text { Lung } \mathrm{CT} \text {, virus nucleic } \\
\text { acid testing, rate of } \\
\text { conversion to severe } \\
\text { cases, and adverse } \\
\text { events }\end{array}$ \\
\hline $\begin{array}{l}\text { Hu et al. } \\
(2020)\end{array}$ & $\begin{array}{l}\text { Mild/ } \\
\text { moderate }\end{array}$ & $\begin{array}{c}\mathrm{T}: 142(79 / \\
63) \mathrm{C}: 142 \\
(71 / 71)\end{array}$ & $\mathrm{T}: 50.4 \pm 15.2$ C:51.8 \pm 14.8 & $\begin{array}{l}\text { Lianhua Qingwen } \\
\text { capsule }(6 \mathrm{~g}, \mathrm{tid})+ \\
\text { conventional } \\
\text { therapy }\end{array}$ & $\begin{array}{l}\text { Conventional therapy } \\
\text { including oxygen therapy, } \\
\text { antiviral medications, and } \\
\text { symptomatic therapies }\end{array}$ & 14 days & $\begin{array}{l}\text { Lung } \mathrm{CT} \text {, clinical cure } \\
\text { rate, virus nucleic acid } \\
\text { testing, rate of conversion } \\
\text { to severe cases, clinical } \\
\text { symptoms, and adverse } \\
\text { events }\end{array}$ \\
\hline $\begin{array}{l}\text { Yu et al. } \\
\text { (2020) }\end{array}$ & $\begin{array}{l}\text { Mild/ } \\
\text { moderate }\end{array}$ & $\begin{array}{c}\mathrm{T}: 147(82 / \\
65) \mathrm{C}: 148 \\
(89 / 59)\end{array}$ & $\mathrm{T}: 48.27 \pm 9.56 \mathrm{C}: 47.25 \pm 8.67$ & $\begin{array}{l}\text { Lianhua Qingwen } \\
\text { granule }(6 \mathrm{~g}, \text { tid })+ \\
\text { conventional } \\
\text { therapy }\end{array}$ & $\begin{array}{l}\text { Conventional therapy } \\
\text { including abidor tablets, } \\
\text { moxifloxacin tablets, and } \\
\text { ambroxol tablets }\end{array}$ & 7 days & $\begin{array}{l}\text { Lung } \mathrm{CT} \text {, clinical cure } \\
\text { rate, rate of conversion to } \\
\text { severe cases, clinical } \\
\text { symptoms } \\
\text { inflammatory biomarkers, } \\
\text { and adverse events }\end{array}$ \\
\hline $\begin{array}{l}\text { Zhang } \\
\text { et al. } \\
\text { (2020) }\end{array}$ & Moderate & $\begin{array}{c}\mathrm{T}: 80(50 / \\
30) \mathrm{C}: 40 \\
(23 / 17)\end{array}$ & $\mathrm{T}: 53.4 \pm 13.70 \mathrm{C}: 52.0 \pm 14.10$ & $\begin{array}{l}\text { Jinyinhua oral liquid } \\
(60 \mathrm{ml} / \text { time, tid })+ \\
\text { conventional } \\
\text { therapy }\end{array}$ & $\begin{array}{l}\text { Conventional therapy } \\
\text { including interferon- } \alpha \text {, } \\
\text { lopinavir, tonavir tablets, and } \\
\text { symptomatic treatment }\end{array}$ & 10 days & $\begin{array}{l}\text { Rate of conversion to } \\
\text { severe cases, clinical } \\
\text { symptoms, and adverse } \\
\text { events }\end{array}$ \\
\hline
\end{tabular}

therapy of honeysuckle and controls. Control groups used conventional therapy. In each trial, conventional therapy in the treatment group was identical to the control group. Two trial intervention groups were Chinese medicine compound drugs (Pneumonia No. 1 formula and Qingfei Touxie Fuzheng recipe) (Ai et al., 2020; Ding et al., 2020). And the other trials were Chinese patent medicine (Duan et al., 2020; Fu et al., 2020a; Fu et al., 2020b; Hu et al., 2020; Yu et al., 2020; Zhang et al., 2020; Hu et al., 2021). Conventional therapy included oxygen therapy, drugs, and symptomatic therapies. The drugs used in the control group were arbidol, lopinavir, interferon- $\alpha$, and ribavirin. Two trial control groups did not provide specific therapy medicine (Duan et al., 2020; Hu et al., 2021).

\section{Description of Honeysuckle}

The description of honeysuckle in each trial is shown in Table 2. Honeysuckle was used in the dosage formulations of granules (55.55\%) (Ai et al., 2020; Duan et al., 2020; Fu et al., 2020a; Fu et al., 2020b; Yu et al., 2020), decoction (11.11\%) (Ding et al., 2020), oral liquid (22.22\%) (Hu et al., 2020; Zhang et al., 2020), and capsule (11.11\%) (Hu et al., 2021). The component of oral liquid (Hu et al., 2020; Zhang et al., 2020) is only honeysuckle. 
TABLE 2 | The description of honeysuckle in each trial.

\begin{tabular}{|c|c|c|}
\hline References & $\begin{array}{l}\text { Honeysuckle and Chinese } \\
\text { herbal medicine }\end{array}$ & Components \\
\hline Ai et al. (2020) & Pneumonia No.1 formula (granule) & $\begin{array}{l}\text { Honeysuckle } 15 \mathrm{~g} \text {, Lianqiao } 30 \mathrm{~g} \text {, Qingdao } 10 \mathrm{~g} \text {, Huangqi } 45 \mathrm{~g} \text {, Shancigu } 20 \mathrm{~g} \text {, Huangqin } 10 \mathrm{~g} \text {, Daqingye } \\
10 \mathrm{~g} \text {, Chaihu } 5 \mathrm{~g} \text {, Chantui } 10 \mathrm{~g} \text {, Qianhu } 5 \mathrm{~g} \text {, Chuanbeimu } 10 \mathrm{~g} \text {, Zhebeimu } 10 \mathrm{~g} \text {, Wumei } 30 \mathrm{~g} \text {, Xuanshen } 10 \mathrm{~g} \text {, } \\
\text { Fuling } 30 \mathrm{~g} \text {, and Taizishen } 15 \mathrm{~g}\end{array}$ \\
\hline Ding et al. (2020) & $\begin{array}{l}\text { Qingfei Touxie Fuzheng recipe } \\
\text { (decoction) }\end{array}$ & $\begin{array}{l}\text { Honeysuckle } 30 \mathrm{~g} \text {, Lianqiao } 15 \mathrm{~g} \text {, Mahuang } 6 \mathrm{~g} \text {, Shigao } 20 \mathrm{~g} \text {, Kuxingren } 10 \mathrm{~g} \text {, Lugen } 30 \mathrm{~g} \text {, Yiyiren } 30 \mathrm{~g} \text {, } \\
\text { Jiangcan } 10 \mathrm{~g} \text {, Chantui } 10 \mathrm{~g} \text {, Huzhang } 15 \mathrm{~g} \text {, Jianghuang } 10 \mathrm{~g} \text {, Baishaoyao } 10 \mathrm{~g} \text {, Taizishen } 20 \mathrm{~g} \text {, and } \\
\text { Gancao } 15 \mathrm{~g}\end{array}$ \\
\hline Duan et al. (2020) & Jinhua Qinggan granule & $\begin{array}{l}\text { Honeysuckle } 10 \mathrm{~g} \text {, Shigao } 10 \mathrm{~g} \text {, Mahuang } 10 \mathrm{~g} \text {, Kuxingren } 10 \mathrm{~g} \text {, Huangqin } 10 \mathrm{~g} \text {, Lianqiao } 10 \mathrm{~g} \text {, Zhebeimu } \\
10 \mathrm{~g} \text {, Zhimu } 10 \mathrm{~g} \text {, Niubangzi } 10 \mathrm{~g} \text {, Qinghao } 10 \mathrm{~g} \text {, Bohe } 10 \mathrm{~g} \text {, and Gancao10 g }\end{array}$ \\
\hline Fu et al. (2020a) & Toujie Quwen granule & $\begin{array}{l}\text { Honeysuckle } 15 \mathrm{~g} \text {, Lianqiao } 30 \mathrm{~g} \text {, Shancigu } 20 \mathrm{~g} \text {, Huangqin } 10 \mathrm{~g} \text {, Daqingye } 10 \mathrm{~g} \text {, Chaihu } 5 \mathrm{~g} \text {, Qinghao } 10 \mathrm{~g} \text {, } \\
\text { Chantui } 10 \mathrm{~g} \text {, Qianhu } 5 \mathrm{~g} \text {, Chuanbeimu } 10 \mathrm{~g} \text {, Zhebeimu } 10 \mathrm{~g} \text {, Wumei } 30 \mathrm{~g} \text {, Xuanshen } 10 \mathrm{~g} \text {, Huangqi } 45 \mathrm{~g} \text {, } \\
\text { Fuling } 30 \mathrm{~g} \text {, and Taizishen } 15 \mathrm{~g}\end{array}$ \\
\hline Fu et al. (2020b) & Toujie Quwen granule & $\begin{array}{l}\text { Honeysuckle } 15 \mathrm{~g} \text {, Lianqiao } 30 \mathrm{~g} \text {, Shancigu } 20 \mathrm{~g} \text {, Huangqin } 10 \mathrm{~g} \text {, Daqingye } 10 \mathrm{~g} \text {, Chaihu } 5 \mathrm{~g} \text {, Qinghao } 10 \mathrm{~g} \text {, } \\
\text { Chantui } 10 \mathrm{~g} \text {, Qianhu } 5 \mathrm{~g} \text {, Chuanbeimu } 10 \mathrm{~g} \text {, Zhebeimu } 10 \mathrm{~g} \text {, Wumei } 30 \mathrm{~g} \text {, Xuanshen } 10 \mathrm{~g} \text {, Huangqi } 45 \mathrm{~g} \text {, } \\
\text { Fuling } 30 \mathrm{~g} \text {, and Taizishen } 15 \mathrm{~g}\end{array}$ \\
\hline Hu et al. (2020) & Jinyinhua oral liquid & Honeysuckle $10.8 \mathrm{~g}$ \\
\hline Hu et al. (2020) & Lianhua Qingwen capsule & $\begin{array}{l}\text { Honeysuckle, Lianqiao, Mahuang, Kuxingren, Shigao, Banlangen, Mianma, Guanzhong, Yuxingcao, } \\
\text { Huoxiang, Dahuang, Hongjingtian, menthol, and Gancao }\end{array}$ \\
\hline Yu et al. (2020) & Lianhua Qingwen granule & $\begin{array}{l}\text { Honeysuckle, Lianqiao, Mahuang, Kuxingren, Shigao, Banlangen, Mianma, Guanzhong, Yuxingcao, } \\
\text { Huoxiang, Dahuang, Hongjingtian, menthol, and Gancao }\end{array}$ \\
\hline Zhang et al. (2020) & Jinyinhua oral liquid & Honeysuckle $5.4 \mathrm{~g}$ \\
\hline
\end{tabular}

TABLE 3 | Risk of bias.

\begin{tabular}{|c|c|c|c|c|c|c|c|}
\hline References & A & B & C & D & $\mathbf{E}$ & $\mathbf{F}$ & G \\
\hline Ai et al. (2020) & L & U & U & U & L & U & U \\
\hline Ding et al. (2020) & $\mathrm{L}$ & $U$ & $U$ & $U$ & $\mathrm{~L}$ & U & $U$ \\
\hline Duan et al. (2020) & $\mathrm{L}$ & $U$ & $U$ & $U$ & $L$ & U & $U$ \\
\hline Fu et al. (2020a) & $\mathrm{L}$ & $U$ & $U$ & $U$ & $\mathrm{~L}$ & U & U \\
\hline Fu et al. (2020b) & $\mathrm{L}$ & $U$ & $U$ & $U$ & $\mathrm{~L}$ & U & $U$ \\
\hline Hu et al. (2020) & $\mathrm{L}$ & $U$ & $U$ & $U$ & $\mathrm{~L}$ & U & $U$ \\
\hline Hu et al. (2020) & $\mathrm{L}$ & $U$ & $U$ & $\mathrm{~L}$ & $\mathrm{~L}$ & U & U \\
\hline Yu et al. (2020) & $\mathrm{L}$ & $U$ & $U$ & $U$ & $\mathrm{~L}$ & U & $U$ \\
\hline Zhang et al. (2020) & U & $U$ & U & U & $L$ & U & $U$ \\
\hline
\end{tabular}

$A$, random sequence generation (selection bias); $B$, allocation concealment (selection bias); $C$, blinding of participants and personnel (performance bias); $D$, blinding of outcome assessment (detection bias); $E$, incomplete outcome data (attrition bias); $F$, selective reporting (reporting bias); $G$, other biases; L, low risk; $H$, high risk; $U$, unclear.

Honeysuckle is one component of Chinese herbal medicine in other dosage formulations.

\section{METHODOLOGICAL QUALITY}

The results of the ROB assessment are shown in Table 3. In general, there was insufficient information available in all trials included in this study. The risks of bias of included trials were mostly "unclear risk."

\section{Results of the Meta-Analysis}

Four trials (Ding et al., 2020; Duan et al., 2020; Hu et al., 2020; Yu et al., 2020) reported lung CT. Compared with conventional therapy alone, combination therapy of honeysuckle and conventional therapy exhibited a significant improvement on lung CT [ 4 trials, $n=744, \mathrm{RR}=1.24,95 \% \mathrm{CI}(1.12,1.37), I^{2}=11 \%$, $p<0.0001]$ (Figure 2A). Subgroup analysis revealed that combination therapy could better improve the lung CT of nonsevere COVID-19 [ 3 trials, $n=644, \mathrm{RR}=1.22,95 \% \mathrm{CI}$ (1.10, 1.35), $\left.I^{2}=25 \%, p=0.0002\right]$ (Figure 2A).

Five trials (Ai et al., 2020; Fu et al., 2020a; Fu et al., 2020b; Yu et al., 2020; Hu et al., 2021) demonstrated the clinical cure rate. The results showed that clinical cure rate in the combination treatment groups was higher than the control groups [ 5 trials, $n=$ $\left.815, \mathrm{RR}=1.21,95 \% \mathrm{CI}(1.12,1.31), I^{2}=19 \%, p<0.00001\right]$ (Figure 2B).

Three trials (Hu et al., 2020; Zhang et al., 2020; Hu et al., 2021) described viral nucleic acid testing. Compared with the control groups, no statistical difference on viral nucleic acid testing was identified [ 3 trials, $n=532, \mathrm{RR}=1.06,95 \% \mathrm{CI}(0.98,1.15), I^{2}=0 \%$, $p=0.15$ ] (Figure 3A). Subgroup analysis suggested no statistical difference between honeysuckle alone $(p=0.32)$ and Chinese herbal medicine formula ( $p=0.28$ ) (Figure 3A).

Six trials (Fu et al., 2020a; Fu et al., 2020b; Hu et al., 2020; Yu et al., 2020; Zhang et al., 2020; Hu et al., 2021) reported rate of conversion to severe cases. We found that combination therapy could significantly reduce the rate of conversion to severe cases [ 6 trials, $n=965, \mathrm{RR}=0.50,95 \% \mathrm{CI}$ (0.33, 0.76), $I^{2}=0 \%, p=0.001$ ] (Figure 3B). Subgroup analysis showed that there was a significant difference between honeysuckle alone $(p=0.04)$ and Chinese herbal medicine formula $(p=0.01)$ (Figure 3B).

Six trials (Ding et al., 2020; Duan et al., 2020; Fu et al., 2020a; Fu et al., 2020b; Yu et al., 2020; Zhang et al., 2020) described clinical symptoms of fever, cough, and fatigue. Meta-analyses revealed that combination therapy could better improve the symptoms reduction rate and symptom score than conventional therapy (Table 4). As shown in Table 4, combination therapy could significantly improve the symptom score of fever, cough reduction rate, symptom score of cough, and symptom score of fatigue $(p<0.05)$. However, there was no significant difference in 


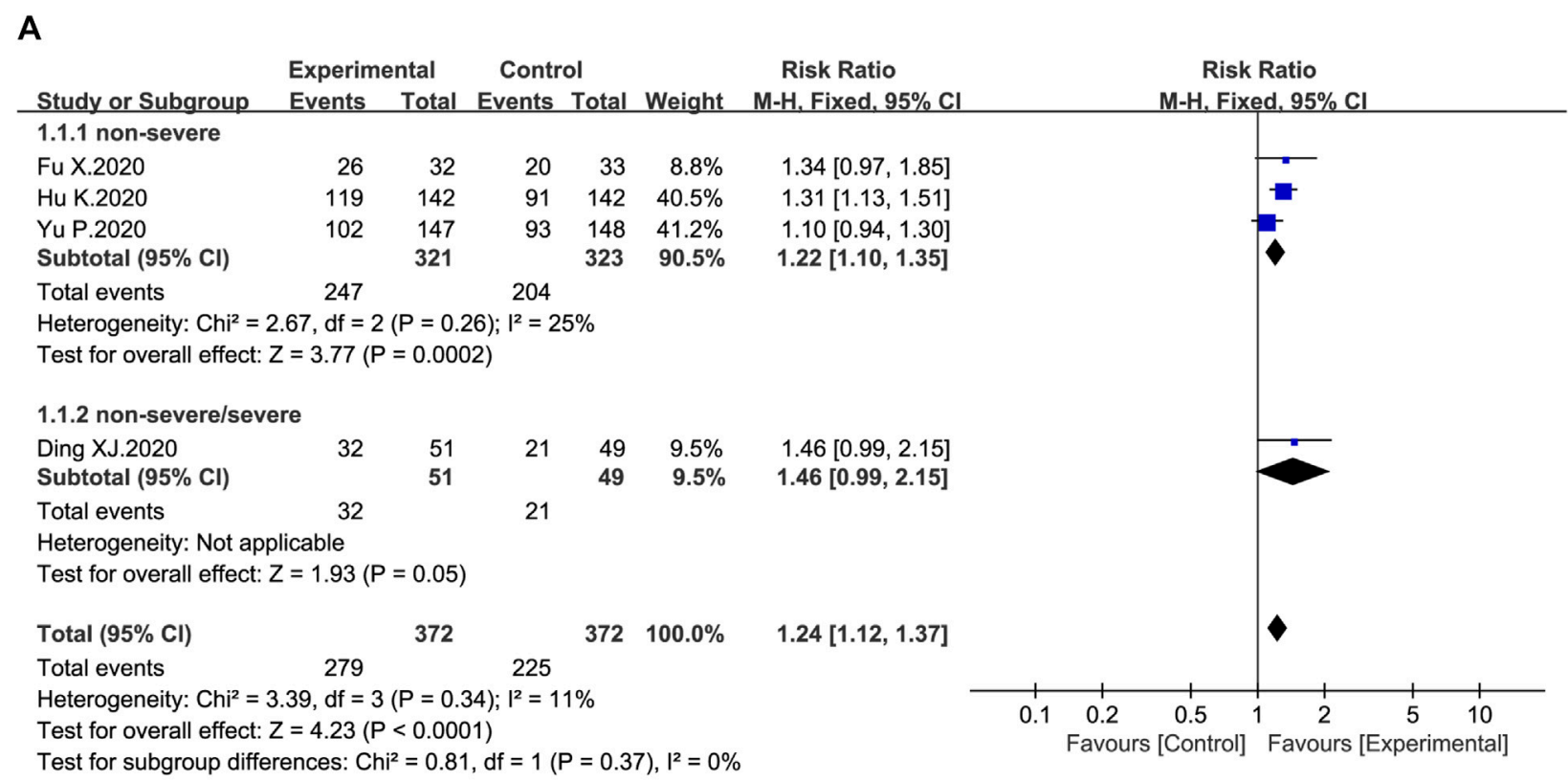

lung CT.

B

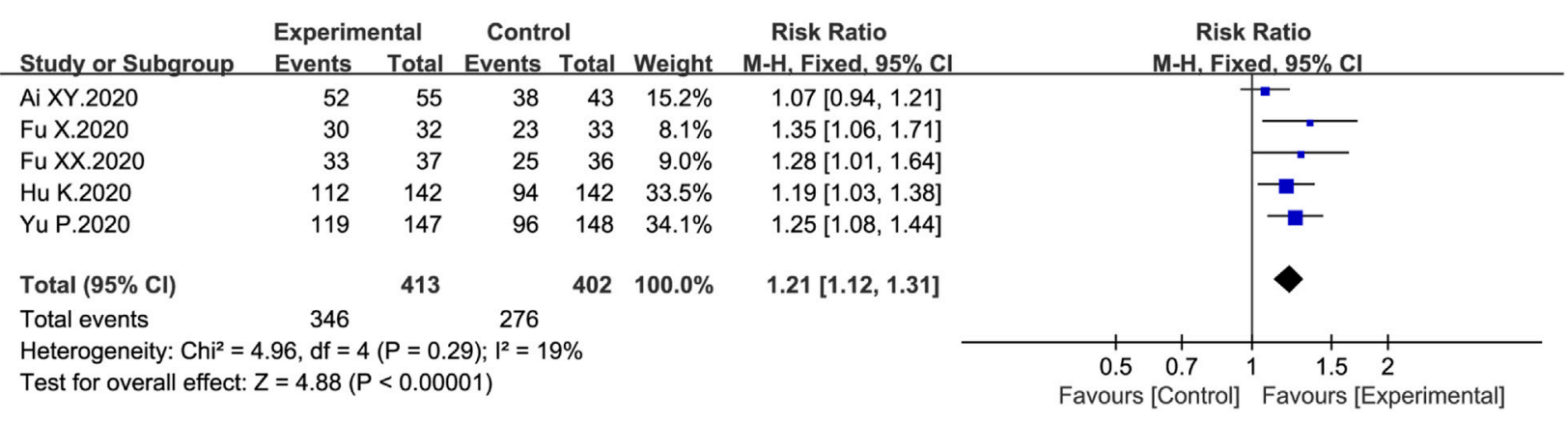

clinical cure rate.

FIGURE 2 | Forest plot of the effects of combination therapy for outcomes of (A) lung CT and (B) clinical cure rate.

fever reduction rate and fatigue reduction rate between the combination treatment and control groups $(p>0.05)$.

Five trials (Ai et al., 2020; Ding et al., 2020; Fu et al., 2020a; Fu et al., 2020b; Yu et al., 2020) reported inflammatory biomarkers. We found that combination therapy was beneficial for WBC count [ 3 trials, $n=433, \mathrm{MD}=0.38,95 \% \mathrm{CI}(0.31,0.44), I^{2}=22 \%$, $p<0.00001$ ], LYM count [ 4 trials, $n=531, \mathrm{MD}=0.23,95 \% \mathrm{CI}$ $(0.05,0.41), I^{2}=97 \%, p=0.01$ ], and CRP level [ 4 trials, $n=533$, $\left.\mathrm{MD}=-12.95,95 \% \mathrm{CI}(-21.18,-4.01), I^{2}=98 \%, p=0.004\right]$ to return to normal. And these differences were statistically significant $(p<0.05)$ (Table 5).

All included trials reported adverse drug events. The common adverse drug events of combination therapy were nausea and vomiting, inappetence, diarrhea, headache, renal dysfunction, and abnormal liver function. As shown in Table 6, there was no significant difference in adverse events rate, diarrhea, and abnormal liver function between the combination treatment and control groups $(p>0.05)$. Additionally, inappetence, nausea and vomiting, headache, and renal dysfunction were reported in one trial ( $\mathrm{Hu}$ et al., 2021), and no statistical difference was identified in both combination treatment and control groups.

\section{Sensitivity Analysis}

Sensitivity analysis revealed a small change in the effect amount, and a significant difference in lung CT, clinical cure rate, rate of conversion to severe cases, symptom score of fever, cough reduction rate, symptom score of cough, WBC count, and CRP. Sensitivity analysis indicated that the above results were robust.

\section{Publication Bias}

As the number of trials in any comparative outcome measure was less than ten, we did not assess the publication bias. 
A

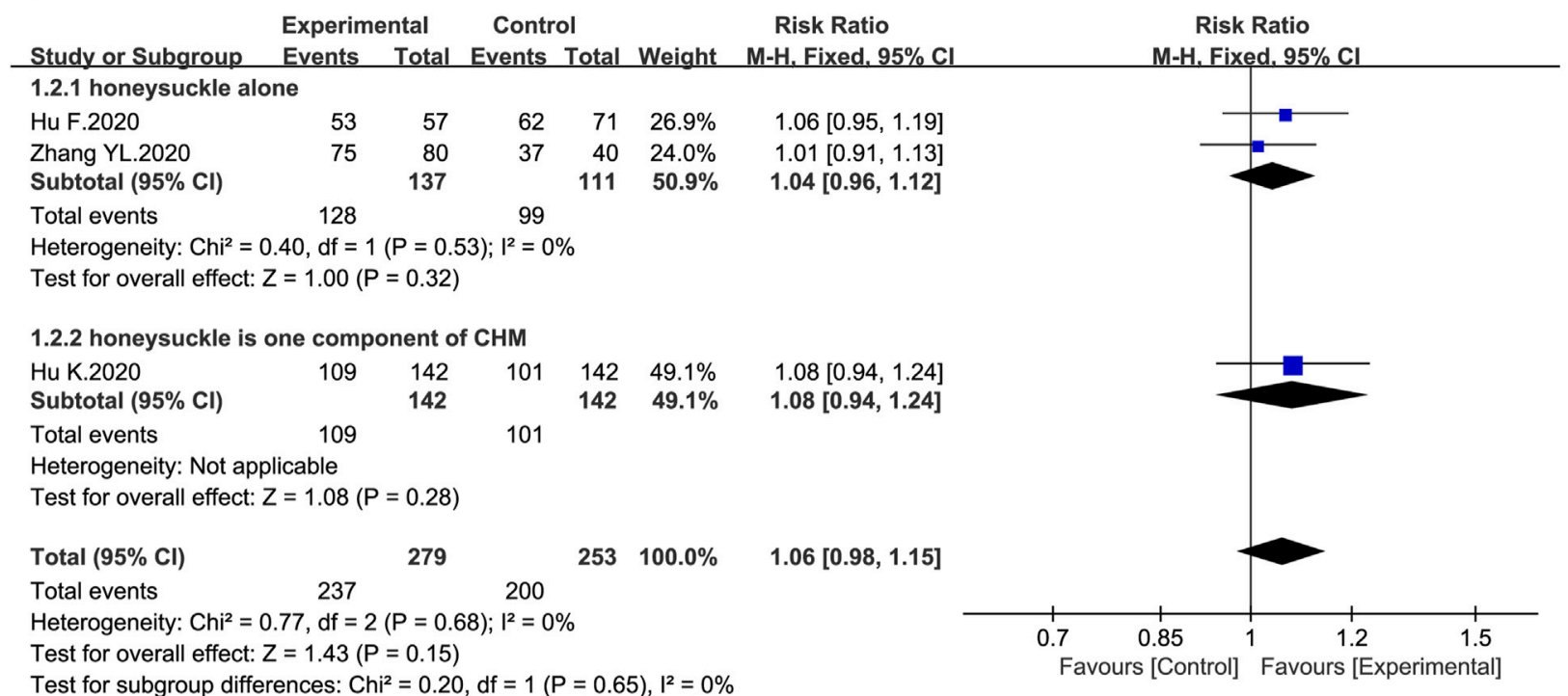

viral nucleic acid testing.

$\mathbf{B}$

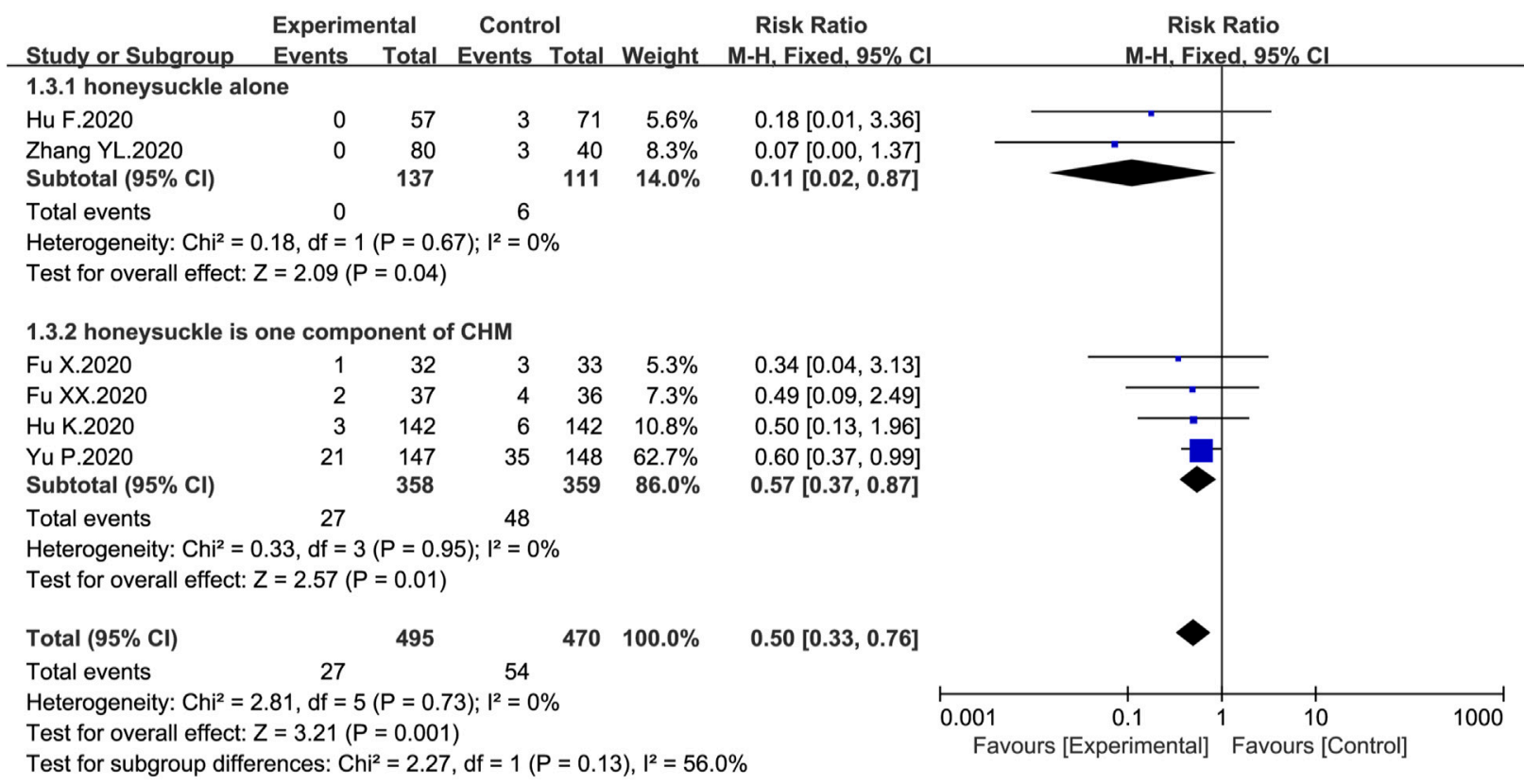

rate of conversion to severe cases.

FIGURE 3 | Forest plot of the effects of combination therapy for outcomes of (A) viral nucleic acid testing and (B) rate of conversion to severe cases.

\section{DISCUSSION}

\section{Summary of Findings}

In our study, it was found that Chinese herbal medicine honeysuckle could provide additional benefit for the clinical outcomes of COVID-19. This finding was consistent with similar studies (Ang et al., 2020; Xiong et al., 2020). Similar studies have shown that, compared with conventional therapy, Chinese herbal medicine had better effects and fewer adverse drug events (Ang et al., 2020; Xiong et al., 2020). Facing such a severe COVID-19 epidemic, Western countries should pay high attention to the curative effect of Chinese herbal medicine.

Honeysuckle is one of the most widely used traditional Chinese herbal medicines. It is used as an antiviral, 
TABLE 4 | Comparison of clinical symptoms between the treatment group and control group.

\begin{tabular}{|c|c|c|c|c|c|c|c|}
\hline \multirow[t]{2}{*}{ Outcome measure } & \multirow{2}{*}{$\begin{array}{c}\text { No. of } \\
\text { trials }\end{array}$} & \multicolumn{3}{|c|}{ Samples } & \multirow[t]{2}{*}{ Statistical method } & \multirow[t]{2}{*}{ Effect estimate } & \multirow[t]{2}{*}{$p$-value } \\
\hline & & Total & Events/intervention & Events/control & & & \\
\hline Fever cases & 3 & 296 & $171 / 185$ & $90 / 111$ & $\mathrm{RR}$ (random) 95\%Cl & $0.11(-0.10,0.33)$ & 0.31 \\
\hline Cough cases & 3 & 260 & $135 / 167$ & $56 / 93$ & $\mathrm{RR}$ (fixed) 95\%Cl & $1.37(1.15,1.65)$ & 0.0006 \\
\hline Fatigue cases & 2 & 163 & $98 / 116$ & $32 / 47$ & $\mathrm{RR}$ (random) 95\%Cl & $1.20(0.85,1.69)$ & 0.3 \\
\hline Symptom score of fever & 3 & 433 & - & - & $\mathrm{RR}$ (random) 95\%Cl & $-0.62(-0.79,-0.45)$ & $<0.00001$ \\
\hline symptom score of cough & 3 & 433 & - & - & $\mathrm{RR}$ (random) 95\%Cl & $-1.18(-1.34,-1.03)$ & $<0.00001$ \\
\hline Symptom score of fatigue & 3 & 433 & - & - & $\mathrm{RR}$ (random) 95\%Cl & $-0.60(-1.04,-0.17)$ & 0.007 \\
\hline
\end{tabular}

TABLE 5 | Comparison of inflammatory biomarkers between the treatment group and control group.

\begin{tabular}{|c|c|c|c|c|c|}
\hline Outcome measure & $\begin{array}{l}\text { No. of } \\
\text { trials }\end{array}$ & Samples & Statistical method & Effect estimate & $p$-value \\
\hline WBC count & 3 & 433 & MD (fixed) 95\%Cl & $0.38(0.31,0.44)$ & $<0.00001$ \\
\hline LYM count & 4 & 531 & $\mathrm{MD}$ (random) 95\%Cl & $0.23(0.05,0.41)$ & 0.01 \\
\hline CRP & 4 & 533 & $\mathrm{MD}$ (random) 95\% Cl & $-12.95(-21.18,-4.01)$ & 0.004 \\
\hline
\end{tabular}

TABLE 6 | Comparison of adverse drug events between the treatment group and control group.

\begin{tabular}{|c|c|c|c|c|c|c|c|}
\hline \multirow[t]{2}{*}{ Outcome measure } & \multirow{2}{*}{$\begin{array}{l}\text { No. of } \\
\text { trials }\end{array}$} & \multicolumn{3}{|c|}{ Samples } & \multirow[t]{2}{*}{ Statistical method } & \multirow[t]{2}{*}{ Effect estimate } & \multirow[t]{2}{*}{$p$-value } \\
\hline & & Total & Events/intervention & Events/control & & & \\
\hline Adverse events rate & 5 & 755 & $96 / 412$ & $80 / 343$ & $\mathrm{RR}$ (random) 95\%Cl & $1.72(0.44,6.74)$ & 0.44 \\
\hline Diarrhea & 4 & 655 & $37 / 361$ & $19 / 294$ & $\mathrm{RR}$ (random) 95\%Cl & $2.43(0.20,29.33)$ & 0.49 \\
\hline Abnormal liver function & 2 & 384 & $34 / 193$ & $35 / 191$ & $\mathrm{RR}$ (fixed) $95 \% \mathrm{Cl}$ & $0.97(0.64,1.47)$ & 0.88 \\
\hline
\end{tabular}

immunomodulator, anti-inflammatory, hepatoprotectant, and nephroprotectant (Miao et al., 2019; Bai et al., 2020; Fang et al., 2020; Alekhya Sita et al., 2019). Honeysuckle is predicted to suppress SARS-CoV-2 replication (Lee et al., 2021). Honeysuckle extracts can inhibit the replication of influenza viruses $\mathrm{H} 1 \mathrm{~N} 1, \mathrm{H} 3 \mathrm{~N} 2$, and the oseltamivir-resistant mutant strain H1N1-H275Y (Li et al., 2020). Honeysuckle polysaccharides can regulate nonspecific immunity (Zhou et al., 2018) and inhibit the expression of inflammatory factors TNF- $\alpha$ and IL-1 $\beta$ (Bai et al., 2020). Neochlorogenic acid from Lonicera can prevent excessive macrophagemediated responses associated with acute and chronic inflammatory disorders (Park et al., 2018). Lonicera caerulea L. polyphenols (LCPs) can alleviate LPS-induced liver injury by suppressing the nuclear translocation of NF- $\mathrm{B}$ p65 and activation of the MAPK signaling pathway (Li et al., 2020). Luteolin is a pharmacologically active component normally found in honeysuckle, which exhibits antioxidant activity and nephroprotective activity (Alekhya Sita et al., 2019).

In our study, honeysuckle combined with conventional therapy was superior to conventional therapy alone in improving clinical symptoms, imaging, and laboratory indexes. Compared with conventional therapy alone, combination therapy of honeysuckle and conventional therapy could improve symptom score of fever, cough reduction rate, and symptom score of cough. We found that combination therapy could improve lung CT, increase WBC count, and reduce CRP level. This is related to the fact that honeysuckle can affect the immune response and production of inflammatory cytokines (Zhou et al., 2018; Bai et al., 2020). Immunopathological changes, including diminished lymphocytes and elevated cytokines, are important drivers of disease progression and death in coronavirus infections (Tang et al., 2020). Cytokine storm is uncontrolled overproduction of inflammation markers with elevated levels of IL-6, IL-1 $\beta$, and TNF- $\alpha$ (Coperchini et al., 2020; Kempuraj et al., 2020). This leads to acute lung injury, acute respiratory distress syndrome (ARDS), and widespread tissue damage resulting in multiorgan failure and death (Ragab et al., 2020; Caricchio et al., 2021). In our study, we also found that combination therapy had improvements in clinical parameters including clinical cure rate and rate of conversion to severe cases.

Safety issues should be another concern when honeysuckle combined with conventional therapy is used for COVID-19 patients. In our study, all included trials reported adverse drug events. Compared with conventional therapy alone, combination therapy of honeysuckle and conventional therapy did not increase adverse drug events. This is related to the fact that honeysuckle exhibits hepatoprotective and nephroprotective activity (Alekhya Sita et al., 2019; Li et al., 2020). 


\section{Strengths and Limitations}

This study is to our knowledge the first systematic review and meta-analysis for the effectiveness and safety of honeysuckle combined with conventional therapy in adult patients with COVID-19. It could help to respond to the current public health emergency. Another advantage could be that only randomized studies are included. Our study was also performed in accordance with Cochrane Handbook and PRISMA checklist to draw quantitative conclusions with scientific and rigorous methods. In addition, we conducted a subgroup analysis and sensitivity analysis. It meant that our meta-analysis results were more robust.

However, our review had several limitations. Merger statistical analysis of some outcomes had unexplained heterogeneity. Most of the included trials had deficiencies in methodology design, including hidden allocation, blinding, and selective reporting. Publication bias was unclear. The drugs used in the control group were different. However, we did not perform subgroup analyses. The treatment duration ranged from 5 to 15 days. We also did not perform subgroup analyses according to treatment duration.

\section{CONCLUSION}

In conclusion, honeysuckle combined with conventional therapy may be beneficial for the treatment of COVID-19 in improving clinical symptoms, lung CT, laboratory indicators, and clinical cure rate and reducing the rate of conversion to severe cases. Besides, combination therapy did not increase adverse drug

\section{REFERENCES}

Ai, X., Luo, C., Lin, L., Xie, M., Fan, H., and Tan, X. (2020). Therapeutic effect of integrated traditional Chinese and western medicine on COVID-19 in Guangzhou. Chin Trop. Med 20, 746-750. doi:10.13604/j.cnki.46-1064/ r.2020.08.12

Alekhya Sita, G. J., Gowthami, M., Srikanth, G., Krishna, M. M., Rama Sireesha, K., Sajjarao, M., et al. (2019). Protective role of luteolin against bisphenol A-induced renal toxicity through suppressing oxidative stress, inflammation, and upregulating Nrf2/ARE/HO-1 pathway. IUBMB Life 71, 1041-1047. doi:10.1002/iub.2066

Ang, L., Song, E., Lee, H. W., and Lee, M. S. (2020). Herbal medicine for the treatment of coronavirus disease 2019 (COVID-19): A Systematic Review and Meta-Analysis of Randomized Controlled Trials. J. Clin. Med. 9, 1583. doi:10.3390/jcm9051583

Bai, X., Chai, Y., Shi, W., Li, Y., Zhang, T., and Liu, P. (2020). Lonicera japonica polysaccharides attenuate ovalbumin-induced allergic rhinitis by regulation of Th17 cells in BALB/c mice. J. Funct. Foods 65, 103758. doi:10.1016/ j.jff.2019.103758

Caricchio, R., Gallucci, M., Dass, C., Zhang, X., Gallucci, S., Fleece, D., et al. (2021). Preliminary predictive criteria for COVID-19 cytokine storm. Ann. Rheum. Dis. 80, 88-95. doi:10.1136/annrheumdis-2020-218323

Coperchini, F., Chiovato, L., Croce, L., Magri, F., and Rotondi, M. (2020). The cytokine storm in COVID-19: An overview of the involvement of the chemokine/chemokine-receptor system. Cytokine Growth Factor. Rev. 53, 25-32. doi:10.1016/j.cytogfr.2020.05.003

Ding, X., Zhang, Y., He, D., Zhang, M., Tan, Y., Yu, A., et al. (2020). Clinical effect and mechanism of Qingfei Touxie Fuzheng recipe in the treatment of COVID19. Herald Med. 39, 640-644. doi:10.3870/j.issn.1004-0781.2020.05.012 events. However, considering the poor methodology of included trials, more high-quality trials are needed to evaluate the efficacy of honeysuckle in the treatment of COVID-19 in the future.

\section{AUTHOR CONTRIBUTIONS}

L-PS and W-FC designed the study. L-PS and X-QD searched databases, collected the data, assessed the quality of study, and performed data analysis. L-PS, X-QD, W-FC, Z-WC, BZ, and $\mathrm{J}-\mathrm{YH}$ contributed to the rationalization of the results. L-PS, $\mathrm{X}-\mathrm{QD}$, and $\mathrm{W}-\mathrm{FC}$ wrote the manuscript.

\section{FUNDING}

This study was funded by the National Natural Science Foundation of China (No. 81573860), Chongqing Medical University Postdoctoral Foundation (No. R11004), and Chongqing Postdoctoral Special Foundation (Yuren Social Office (2020) No. 379). The funding source had no role in the study.

\section{SUPPLEMENTARY MATERIAL}

The Supplementary Material for this article can be found online at: https://www.frontiersin.org/articles/10.3389/fphar.2021.708636/ full\#supplementary-material

Duan, C., Xia, G., Zheng, C., Sun, G., Li, Z., Li, Q., et al. (2020). Clinical observation on Jinhua Qinggan granule combined with conventional western medicine therapy in treating mild cases of coronavirus disease 2019. J. Tradit Chin. Med. 61, 1473-1477. doi:10.13288/j.11-2166/r.2020.17.001

Fang, Z., Li, J., Yang, R., Fang, L., and Zhang, Y. (2020). A Review: The triterpenoid saponins and biological activities of lonicera linn. Molecules 25, 3773. doi:10.3390/molecules25173773

Fu, X., Lin, L., and Tan, X. (2020a). Clinical observation on effect of Toujie Quwen granules in treatment of COVID-19. Chin. J. Exp. Tradit Med. Formul 26, 44-48. doi:10.13422/j.cnki.syfjx.20201314

Fu, X., Lin, L., and Tan, X. (2020b). Clinical study on 37 case of COVID- 19 treated with integrated traditional Chinese and western medicine. Tradit Chin. Drug Res. Clin. Pharmacol. 31, 600-604. doi:10.19378/j.issn.1003-9783.2020.05.016

Higgins, J. P. T., and Green, S. (2014). Cochrane Reviewers' Handbook 5.3.0. (updated March 2014). Cochrane Collaboration website. Available: www. cochrane-handbook.org (Accessed May 10, 2021).

Hu, F., Guo, A., Huang, L., Yu, W., Liu, G., Gao, X., et al. (2020). Multi-center clinical observation of Jinyinhua oral liquid combined with western medicine in treatment of moderate COVID-19. J. Tradit Chin. Med.. Available online at: https://kns.cnki.net/kcms/detail/11.2166.R.20200819.0837.002.html (Accessed May 2, 2021).

Hu, K., Guan, W.-j., Bi, Y., Zhang, W., Li, L., Zhang, B., et al. (2021). Efficacy and safety of Lianhuaqingwen capsules, a repurposed Chinese herb, in patients with coronavirus disease 2019: A multicenter, prospective, randomized controlled trial. Phytomedicine 85, 153242. doi:10.1016/ j.phymed.2020.153242

Kempuraj, D., Selvakumar, G. P., Ahmed, M. E., Raikwar, S. P., Thangavel, R., Khan, A., et al. (2020). COVID-19, mast cells, cytokine storm, psychological stress, and neuroinflammation. Neuroscientist 26, 402-414. doi:10.1177/ 1073858420941476 
Lee, Y.-R., Chang, C.-M., Yeh, Y.-C., Huang, C.-Y., Lin, F.-M., Huang, J.-T., et al. (2021). Honeysuckle aqueous extracts induced let-7a suppress EV71 replication and pathogenesis in vitro and in vivo and is predicted to inhibit SARS-CoV-2. Viruses 13, 308. doi:10.3390/v13020308

Li, B., Cheng, Z., Sun, X., Si, X., Gong, E., Wang, Y., et al. (2020). Lonicera caerulea L. Polyphenols alleviate oxidative stress-induced intestinal environment imbalance and lipopolysaccharide-induced liver injury in HFD-fed rats by regulating the Nrf2/HO-1/NQO1 and MAPK pathways. Mol. Nutr. Food Res. 64, e1901315. doi:10.1002/mnfr.201901315

Li, M., Wang, Y., Jin, J., Dou, J., Guo, Q., Ke, X., et al. (2020). Inhibitory activity of honeysuckle extracts against influenza A virus in vitro and in vivo. Virol. Sin. 36, 490-500. doi:10.1007/s12250-020-00302-6

Li, Y., Li, W., Fu, C., Song, Y., and Fu, Q. (2020). Lonicerae japonicae flos and Lonicerae flos: a systematic review of ethnopharmacology, phytochemistry and pharmacology. Phytochem. Rev. 19, 1-61. doi:10.1007/s11101-019-09655-7

Liu, M., Gao, Y., Yuan, Y., Yang, K., Shi, S., Zhang, J., et al. (2020). Efficacy and safety of integrated traditional Chinese and western medicine for corona virus disease 2019 (COVID-19): a systematic review and meta-analysis. Pharmacol. Res. 158, 104896. doi:10.1016/j.phrs.2020.104896

Miao, H., Zhang, Y., Huang, Z., Lu, B., and Ji, L. (2019). Lonicera japonica attenuates carbon tetrachloride-induced liver fibrosis in mice: molecular mechanisms of action. Am. J. Chin. Med. 47, 351-367. doi:10.1142/ S0192415X19500174

Moher, D., Liberati, A., Tetzlaff, J., and Altman, D. G.PRISMA Group (2009). Preferred reporting items for systematic reviews and meta-analyses: the PRISMA statement. BMJ 339, b2535-W64. doi:10.7326/0003-4819-151-4200908180-0013510.1136/bmj.b2535

National Health Commission of the people's Republic of China (2021). Epidemic notification. Available: http://www.nhc.gov.cn/xcs/xxgzbd/gzbd_index. shtml2021 (Accessed May 7, 2021).

National Health Commission of the people's Republic of ChinaNational Administration of Traditional Chinese Medicine (2020). Diagnosis and Treatment Guideline for COVID-19 (Trial 8th Edition)]. Chin. J. Viral Dis. 10, 321-328. doi:10.16505/j.2095-0136.2020.0071

Panahi, Y., Hosseini, M. S., Khalili, N., Naimi, E., Majeed, M., and Sahebkar, A. (2015). Antioxidant and anti-inflammatory effects of curcuminoid-piperine combination in subjects with metabolic syndrome: A randomized controlled trial and an updated meta-analysis. Clin. Nutr. 34, 1101-1108. doi:10.1016/ j.clnu.2014.12.019

Park, S. Y., Jin, M. L., Yi, E. H., Kim, Y., and Park, G. (2018). Neochlorogenic acid inhibits against LPS-activated inflammatory responses through up-regulation of Nrf2/HO-1 and involving AMPK pathway. Environ. Toxicol. Pharmacol. 62, 1-10. doi:10.1016/j.etap.2018.06.001

Ragab, D., Salah Eldin, H., Taeimah, M., Khattab, R., and Salem, R. (2020). The COVID-19 cytokine storm; what we know so far. Front. Immunol. 11, 1446. doi:10.3389/fimmu.2020.01446
Sattler, A., Angermair, S., Stockmann, H., Heim, K. M., Khadzhynov, D., Treskatsch, S., et al. (2020). SARS-CoV-2-specific T cell responses and correlations with COVID-19 patient predisposition. J. Clin. Invest. 130, 6477-6489. doi:10.1172/JCI140965

Tang, L., Yin, Z., Hu, Y., and Mei, H. (2020). Controlling cytokine storm is vital in COVID-19. Front. Immunol. 11, 570993. doi:10.3389/ fimmu.2020.570993

World Health Organization (WHO) (2021). WHO Coronavirus (COVID-19) Dashboard. Available: https://covid19.who.int/2021 (Accessed May 7, 2021).

Xiong, X., Wang, P., Su, K., Cho, W. C., and Xing, Y. (2020). Chinese herbal medicine for coronavirus disease 2019: A systematic review and meta-analysis. Pharmacol. Res. 160, 105056. doi:10.1016/j.phrs.2020.105056

Yu, P., Li, Y., Wan, S., and Wang, Y. (2020). Clinical efficacy of Lianhua Qingwen granule combined with abidol in treating coronavirus disease 2019. Chin. Pharm. J. 55, 1042-1045. Available online at: http://kns.cnki. net/kcms/detail/11.2162.R.20200422.1429.002.html (Accessed May 2, 2021).

Zhang, Y., Lei, L., Xu, Y., Wei, D., and Hu, F. (2020). Clinical efficacy of Jinyinhua oral liquid in the treatment of 80 patients with coronavirus disease 2019. Chin. Pharm. 29, 23-26. doi:10.3969/j.issn.1006-4931.2020.09.006

Zhou, L. K., Zhou, Z., Jiang, X. M., Zheng, Y., Chen, X., Fu, Z., et al. (2020). Absorbed plant MIR2911 in honeysuckle decoction inhibits SARS-CoV-2 replication and accelerates the negative conversion of infected patients. Cell Discov 6, 54. doi:10.1038/s41421-020-00197-3

Zhou, X., Dong, Q., Kan, X., Peng, L., Xu, X., Fang, Y., et al. (2018). Immunomodulatory activity of a novel polysaccharide from Lonicera japonica in immunosuppressed mice induced by cyclophosphamide. PLoS One 13, e0204152-15. doi:10.1371/journal.pone.0204152

Conflict of Interest: The authors declare that the research was conducted in the absence of any commercial or financial relationships that could be construed as a potential conflict of interest.

Publisher's Note: All claims expressed in this article are solely those of the authors and do not necessarily represent those of their affiliated organizations or those of the publisher, the editors, and the reviewers. Any product that may be evaluated in this article or claim that may be made by its manufacturer is not guaranteed or endorsed by the publisher.

Copyright (c) $2021 \mathrm{Du}$, Shi, Cao, Chen, Zuo and Hu. This is an open-access article distributed under the terms of the Creative Commons Attribution License (CC BY). The use, distribution or reproduction in other forums is permitted, provided the original author(s) and the copyright owner(s) are credited and that the original publication in this journal is cited, in accordance with accepted academic practice. No use, distribution or reproduction is permitted which does not comply with these terms. 